\title{
A RECURSIVE PROCESS RELATED TO A PARTIZAN VARIATION OF WYTHOFF $^{1}$
}

\author{
Alda Carvalho \\ High Institute of Engineering of Lisbon \& CEMAPRE-ISEG, Lisboa, Portugal \\ Carlos P. Santos ${ }^{2}$ \\ High Institute of Education and Science, Lisboa $\&$ CIMA-UE, Evora, Portugal \\ carlos.santos@isec.universitas.pt
}

Cátia Lente Dias

High Institute of Engineering of Lisbon 8 CIMA-UE, Evora, Portugal

Francisco Coelho

University of Evora \& LabMAg, Lisboa, Portugal

João Pedro Neto

University of Lisbon \& LabMAg, Lisboa, Portugal

Sandra Vinagre

University of Evora $\&$ CIMA-UE, Evora, Portugal

Received: 8/6/11, Revised: 2/24/12, Accepted: 5/15/12, Published: 5/25/12

\begin{abstract}
WYTHOFF QUEENS is a classical combinatorial game related to very interesting mathematical results. An amazing one is the fact that the $\mathcal{P}$-positions are given by $\left(\lfloor\varphi n\rfloor,\left\lfloor\varphi^{2} n\right\rfloor\right)$ and $\left(\left\lfloor\varphi^{2} n\right\rfloor,\lfloor\varphi n\rfloor\right)$ where $\varphi=\frac{1+\sqrt{5}}{2}$. In this paper, we analyze a different version where one player (Left) plays with a chess bishop and the other (Right) plays with a chess knight. The new game (call it CHESSFIGHTs) lacks a Beatty sequence structure in the $\mathcal{P}$-positions as in WYTHOFF QUEENS. However, it is possible to formulate and prove some general results of a general recursive law which is a particular case of a PARTIZAN SUBTRACTION game. ${ }^{3}$

\footnotetext{
${ }^{1}$ Research in Play mathematics - A Workshop in Combinatorial Game Theory, Center for Mathematics and Fundamental Applications, Lisbon, November, 2010. http://ptmat.fc.ul.pt/arquivo/docs/seminarios/confwork/2010/Mini_workshop.pdf

${ }^{2}$ Correspondent author: Alameda das Linhas de Torres, 179, 1750-142, Lisboa, Portugal

${ }^{3}$ Supported by the research centers CEMAPRE-ISEG, CIMA-UE, LabMAg (Laboratório de Modelação de Agentes), FCT-Portugal funding program.
} 


\section{Introduction}

WYTHOFF QUEENS is played on a quarter-infinite chessboard, extending downwards and to the right. A chess queen is placed in some cell of the board. On each turn, a player moves the queen as in chess, except that the queen can only move left, up, or diagonally up-left. The player who moves the queen to the corner $(0,0)$ wins.

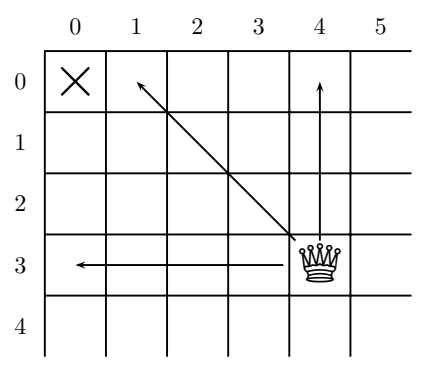

We can also interpret WYTHOFF QUEENS as a pile game. There are two piles of stones and, on each turn, a player either removes an arbitrary number of stones from one pile, or the same number of stones from both piles. The player who makes the last move wins.

A nice result about WYTHOFF QUEENS is the following one (first proved in [6]): The $\mathcal{P}$-positions of WYTHOFF QUEENS are given by $\left(\lfloor\varphi n\rfloor,\left\lfloor\varphi^{2} n\right\rfloor\right)$ and $\left(\left\lfloor\varphi^{2} n\right\rfloor,\lfloor\varphi n\rfloor\right)$ where $\varphi=\frac{1+\sqrt{5}}{2}$.

There are some variations of the game. One very interesting, analyzed in [2] (page 56 ), is the game WHITE KNIGHT. In this variation, instead of a queen, the players move a chess knight. The legal moves are the following (row $x$ and column $y$ ):

$(x, y) \rightarrow(x-1, y-2)$ or $(x, y) \rightarrow(x+1, y-2)$ or $(x, y) \rightarrow(x-2, y-1)$ or $(x, y) \rightarrow(x-2, y+1)$

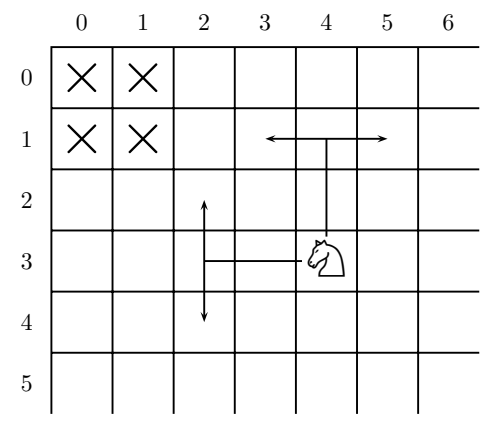

We consider a variation of WYTHOFF QUEENs, the game CHESSFIGHTS. The rules of this variation are the following ones: 
- The board is as in WYTHOFF QUEENS and WhITE KNIGHT;

- Right plays with the knight as in WhITE KNIGHT;

- Left plays with the bishop: $(x, y) \rightarrow(x-i, y-i)$ or $(x, y) \rightarrow(x+i, y-i)$ (in the first case, we must have $x-i \geqslant 0 \wedge y-i \geqslant 0$ and, in the second case, we must have $x+i \geqslant 0 \wedge y-i \geqslant 0$, in other words, the move must be made inside the board).

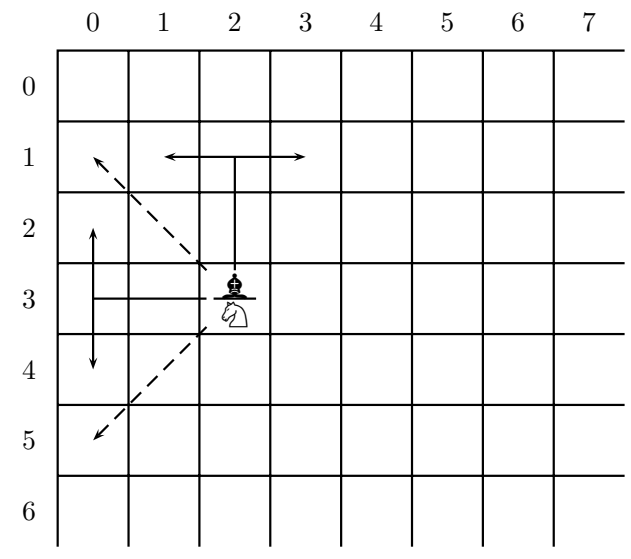

CHESSFIGHTS is a partizan game. For ease, the game with the piece in the cell $(x, y)$ will be represented by the pair $(x, y)$.

The game converges to the end because, after two moves, $(x, y) \mapsto\left(x^{\prime}, y^{\prime}\right) \mapsto$ $\left(x^{\prime \prime}, y^{\prime \prime}\right)$, we have $x^{\prime \prime}+y^{\prime \prime}<x+y$.

\section{Some Theorems of CHESSFIGHTS}

The options of a game are all those positions which can be reached in one move. In combinatorial game theory, games can be expressed recursively as $G=\left\{\mathcal{G}^{L} \mid \mathcal{G}^{R}\right\}$ where $\mathcal{G}^{L}$ are the Left options and $\mathcal{G}^{R}$ are the Right options of $G$. The followers of $G$ are all the games that can be reached by all the possible sequences of moves from $G$ (this is the usual notation of [3], [2], and [1]).

In the particular case of CHESSFIGHTS, we can compute the values of the cells (or, rather, the games corresponding to the placement of a single piece in a cell). The best way to do it is to choose a diagonal path: 


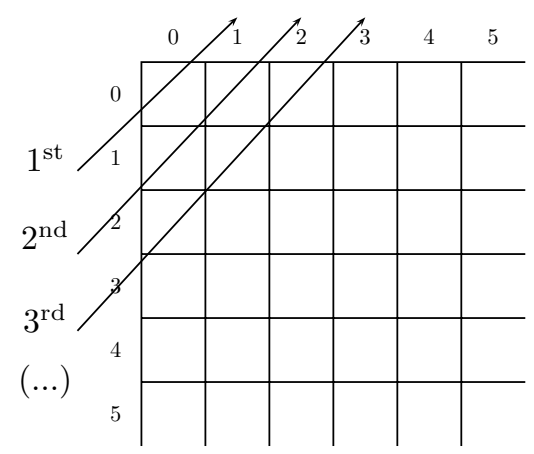

With this procedure, we get an organized table (the following example corresponds to $9 \times 9$ ):

\begin{tabular}{|c|c|c|c|c|c|c|c|c|}
\hline 0 & 1 & $\{1 \mid 0\}$ & $\frac{1}{2}$ & 1 & $\{1 \mid \uparrow\}$ & $\frac{1}{2}$ & 1 & $\{1 \mid \uparrow 3 *\}$ \\
\hline 0 & 1 & $\{1 \mid 0\}$ & $\uparrow$ & 1 & $\left\{1 \mid \frac{1}{2},\{1 \mid *\}\right\}$ & $\uparrow 3 *$ & 1 & $\left\{1 \mid \frac{1}{2}\right\}$ \\
\hline 0 & $*$ & $\{1 \mid 0\}$ & $\{1 \mid *\}$ & $\Uparrow$ & $\{1 \mid \uparrow\}$ & $\{1 \mid 1,\{1 \mid * 2\}\}$ & $\uparrow 3$ & $\{1 \mid \uparrow 3 *\}$ \\
\hline 0 & $*$ & $\uparrow *$ & $\{1 \mid *\}$ & $\{1 \mid * 2\}$ & $\Uparrow \uparrow * 3 \mid \uparrow$ & $\{1 \mid \Uparrow,\{1 \mid * 2\}\}$ & $\{1 \mid\{1 \mid \uparrow\}, \uparrow 3 *\}$ & $\uparrow 3 * 3$ \\
\hline 0 & $*$ & $* 2$ & $\uparrow$ & $\{1 \mid * 2\}$ & $\{1 \mid \uparrow\}$ & $\Uparrow \Uparrow * 2$ & $\{1 \mid \Uparrow *,\{1|| 0 \mid *, * 2\}\}$ & $\{1 \mid \uparrow 3,\{1 \mid \uparrow * 3\}\}$ \\
\hline 0 & $*$ & $* 2$ & $\uparrow$ & $\uparrow * 3$ & $\{1|| 0 \mid *, * 2\}$ & $\{1 \mid \uparrow * 3\}$ & $\{0|| 0 \mid *, * 2\}$ & $\{1 \mid \Uparrow * 2\}$ \\
\hline 0 & $*$ & $* 2$ & $\{0 \mid *, * 2\}$ & $\uparrow * 3$ & $\{0|| 0 \mid *, * 2\}$ & $\{1 \mid \uparrow * 3\}$ & $\{1|| 0|| 0 \mid *, * 2\}$ & $\{0|| 0 \mid * 2,\{0 \mid *, * 2\}\}$ \\
\hline 0 & $*$ & $* 2$ & $\{0 \mid *, * 2\}$ & $\uparrow * 3$ & $\{0|| 0 \mid *, * 2\}$ & $\{0|| 0 \mid * 2,\{0 \mid *, * 2\}\}$ & $\{1|| 0|| 0 \mid *, * 2\}$ & $\{1|| 0|| 0 \mid * 2,\{0 \mid *, * 2\}\}$ \\
\hline 0 & $*$ & $* 2$ & $\{0 \mid *, * 2\}$ & $\{0 \mid * 2,\{0 \mid *, * 2\}\}$ & $\{0|| 0 \mid *, * 2\}$ & $\{0|| 0 \mid * 2,\{0 \mid *, * 2\}\}$ & $\{0|| 0 \mid *, * 2\}$ & $\{1|| 0|| 0 \mid * 2,\{0 \mid *, * 2\}\}$ \\
\hline
\end{tabular}

The same table just with the reduced canonical forms:

\begin{tabular}{|c|c|c|c|c|c|c|c|c|}
\hline 0 & 1 & $\{1 \mid 0\}$ & $\frac{1}{2}$ & 1 & $\{1 \mid 0\}$ & $\frac{1}{2}$ & 1 & $\{1 \mid 0\}$ \\
\hline 0 & 1 & $\{1 \mid 0\}$ & 0 & 1 & $\left\{1 \mid \frac{1}{2}\right\}$ & 0 & 1 & $\left\{1 \mid \frac{1}{2}\right\}$ \\
\hline 0 & 0 & $\{1 \mid 0\}$ & $\{1 \mid 0\}$ & 0 & $\{1 \mid 0\}$ & 1 & 0 & $\{1 \mid 0\}$ \\
\hline 0 & 0 & 0 & $\{1 \mid 0\}$ & $\{1 \mid 0\}$ & 0 & $\{1 \mid 0\}$ & $\{1 \mid 0\}$ & 0 \\
\hline 0 & 0 & 0 & 0 & $\{1 \mid 0\}$ & $\{1 \mid 0\}$ & 0 & $\{1 \mid 0\}$ & $\{1 \mid 0\}$ \\
\hline 0 & 0 & 0 & 0 & 0 & $\{1 \mid 0\}$ & $\{1 \mid 0\}$ & 0 & $\{1 \mid 0\}$ \\
\hline 0 & 0 & 0 & 0 & 0 & 0 & $\{1 \mid 0\}$ & $\{1 \mid 0\}$ & 0 \\
\hline 0 & 0 & 0 & 0 & 0 & 0 & 0 & $\{1 \mid 0\}$ & $\{1 \mid 0\}$ \\
\hline 0 & 0 & 0 & 0 & 0 & 0 & 0 & 0 & $\{1 \mid 0\}$ \\
\hline
\end{tabular}

A visual inspection of the table allows us to guess some patterns. In fact, it is possible to prove some results.

Proposition 1. $(x, 0)=0$.

Proof. Left has no options. Right has no options (cases $(0,0)$ and $(1,0))$ or Right has just one option to $(x-2,1)$. If so, Left plays to $(x-1,0)$ and, by induction, Right loses.

In the next results, it is important to consider the following groups of cells:

$-\operatorname{Red} \longrightarrow(x, y): y-x \equiv 0 \quad(\bmod 3)$ 
- Yellow $\longrightarrow(x, y): y-x \equiv 1(\bmod 3)$

- Green $\longrightarrow(x, y): y-x \equiv 2(\bmod 3)$

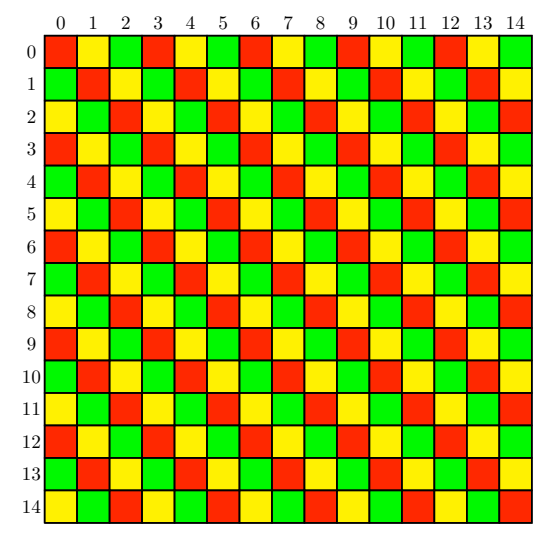

Lemma 1. From the games in the following region (call it $\mathfrak{R}$ ),

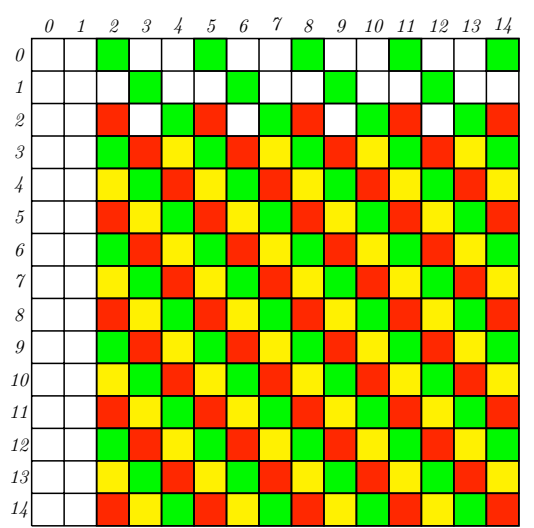

Right to move, has a strategy allowing, at all times, if the sub-position is still not zero, a Right move to a green cell or a Right move to zero.

Proof. Let us analyze all the possible sub-positions $(a, b)$ (Right moving).

- If $b=0$ then the position $(a, b)=0$ (Proposition 1$)$.

- If $(a, b) \in \mathfrak{R}$ is green $(b-a \equiv 2(\bmod 3))$ then Right moves to $(a+1, b-2)$. We can see that $(a+1, b-2)$ remains green because $(b-2)-(a+1) \equiv 2$ $(\bmod 3)$.

- If $(a, b) \in \mathfrak{R}$ is red $(b-a \equiv 0(\bmod 3))$ then Right moves to $(a-1, b-2)$. We can see that $(a-1, b-2)$ turns green because $(b-2)-(a-1) \equiv 2 \quad(\bmod 3)$. 
- If $(a, b) \in \mathfrak{R}$ is yellow $(b-a \equiv 1 \quad(\bmod 3))$ then Right moves $(a-2, b-1)$. We can see that $(a-2, b-1)$ turns green because $(b-1)-(a-2) \equiv 2(\bmod 3)$.

- The only possible Left moves to $(a, b) \notin \Re$ are $(a, 0)$ (item 1$)$ and $(a, 1) \wedge a>1$ (in this case, the Right option to $(a-2, b-1)=0$ is available). The moves indicated in the previous items never allow other options $(a, b) \notin \mathfrak{R}$ for Left.

Proposition 2. $(0,3 k+1)=1(k \geqslant 0)$ and $(0,3 k)=\frac{1}{2}(k \geqslant 1)$.

Proof. Let us prove that $(0,3 k+1)=1(k \geqslant 0)$.

The base case $(0,1)=1$ is calculated by hand. We want to prove that, for $k \geqslant 1$, $(0,3 k+1)+\{\mid 0\}=0$, i.e., $(0,3 k+1)+\{\mid 0\}$ is in $\mathcal{P}$.

If Right plays to $(0,3 k+1)$, Left replies to $(3 k+1,0)=0$ (Proposition 1$)$.

If Right plays to $(1,3 k-1)+\{\mid 0\}$, Left replies to $(0,3 k-2)+\{\mid 0\}=(0,3(k-1)+1)+\{\mid 0\}=1-1$ (induction).

So, if Right plays, Right loses.

If Left plays first to $(a, b)+\{\mid 0\}$ then $(a, b) \in \mathfrak{R}$ or $(a, b)=(a, 0)$ or $(a, b)=$ $(a, 1) \wedge a>1$. The last two cases are trivial. For the first case, Right just plays in $(a, b)$ with the strategy of the Lemma 1 eventually ending in $0-1$. So, playing first, Left loses.

Let us prove that $(0,3 k)=\frac{1}{2}(k \geqslant 1)$. The base case $(0,3)=\frac{1}{2}$ is calculated by hand. We want to prove that, for $k>1,(0,3 k)+\{-1 \mid 0\}=0$, i.e., $(0,3 k)+\{-1 \mid 0\}$ is in $\mathcal{P}$.

If Right plays to $(0,3 k)$, Left replies to $(3 k, 0)=0$ (Proposition 1$)$.

If Right plays to $(1,3 k-2)+\{-1 \mid 0\}$, Left replies to $(0,3 k-3)+\{-1 \mid 0\}=(0,3(k-1))+\{-1 \mid 0\}=\frac{1}{2}-\frac{1}{2}$ (induction).

So, if Right plays, Right loses.

If Left plays first to $(1,3 k-1)+\{-1 \mid 0\}$, Right replies to $(0,3 k-3)+\{-1 \mid 0\}=(0,3(k-1))+\{-1 \mid 0\}=\frac{1}{2}-\frac{1}{2}$ (induction).

If Left plays to $(a, b)+\{-1 \mid 0\}$ with $a>1$ then $(a, b) \in \mathfrak{R}$ or $(a, b)=(a, 0)$ or $(a, b)=(a, 1) \wedge a>1$. The last two cases are trivial. For the first case, Right just plays in $(a, b)$ with the strategy of the Lemma 1 eventually ending in $0-\frac{1}{2}$. So, playing first, Left loses.

The next proposition is a useful inequality. With this result it will be possible to make some arguments of domination and reversibility.

We will write $(x, y)$ to represent the game $(x, y)$, but Left playing with the Knight and Right with the Bishop. We have $-(x, y)=(x, y)$. This is a nice tool to perform 
proofs on the board with two different pieces. Also, we call principal diagonal to the set of cells such that $x=y$.

Lemma 2. If $k \geqslant 2$ and $x^{\prime}>y-k$ then $(x, y)+\left(x^{\prime}, y-k\right) \nless 0$ (if the second component is below the principal diagonal and the components are separated by more than one column, Left wins playing first).

Proof. If $y-k=0$ then $\underline{\left(x^{\prime}, y-k\right)}=0$ (Proposition 1). So, Left plays in the other component to $(x+y, 0) \overline{+\left(x^{\prime}, 0\right)}$ going to zero.

If $y-k=1$, Left moves to $(x, y)+\left(x^{\prime}-2,0\right)$ which is equal to $(x, y)$ (Proposition 1). Following, after a move by Right in $(x, y)$, Left moves this component to the column 0 .

If $y-k>1$, Left moves to $(x, y)+\underline{\left(x^{\prime}+1, y-k-2\right)}$. Following, all the possible moves by Right maintain the Lemma conditions. So, by induction, Left wins.

Proposition 3. If $x>y$ then $(x-k, y) \geqslant(x, y)(k \geqslant 0$, positions inside the board).

Proof. We want to prove that, if $x>y,(x-k, y)-(x, y) \geqslant 0$. So, we want to prove that Right loses playing first in the game $(x-k, y)+(x, y)$. We will analyze all the Right options (consider the principal diagonal, red cells such that $x=y$ ).

- Right plays to $(x-k, y)+\underline{(x+i, y-i)}$. Left moves to $(x-k+i, y \overline{-i)+\underline{(x+i}, y-i)}$ and, by induction, Left wins.
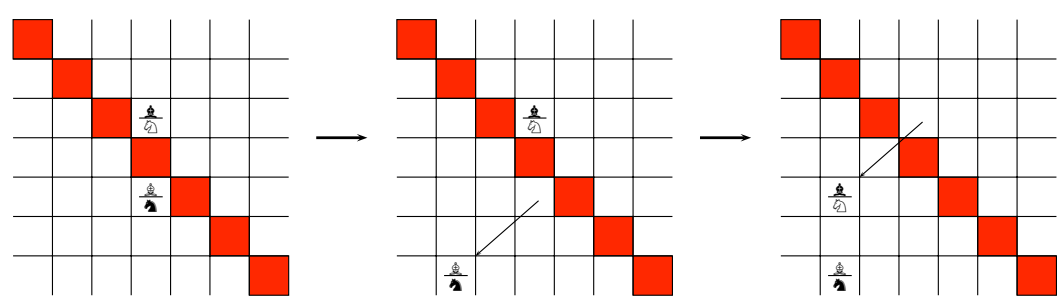

- Right plays to $(x-k, y)+(x-1, y-1)$ (and $\mathrm{k}_{\llcorner} 1$ ).

Left moves to $(x-k+1, y-1)+\underline{(x-1}, y-1)$ and, by induction, Left wins.
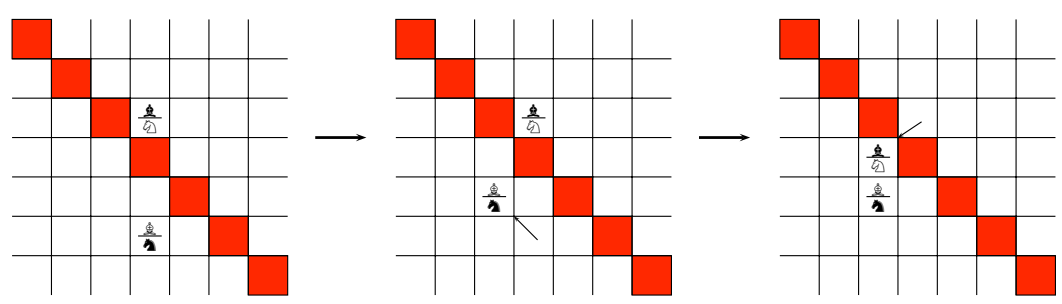

- Right plays to $(x-k, y)+\underline{(x-1, y-1)}$ (and $k \leqslant 1)$.

Left moves to $(x-k-1, y \overline{-1)+\underline{(x-1}, y-1)}$ (available) and, by induction, 
Left wins.

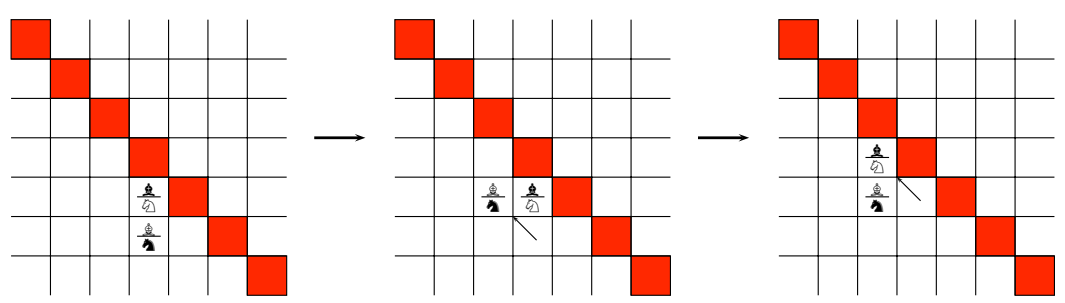

- Right plays to $(x-k, y)+(x-i, y-i)(i>1)$.

By Lemma 2, Left wins.

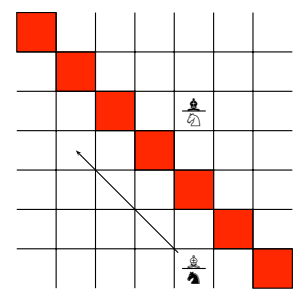

- Right plays to $(x-k+1, y-2)+(x, y)$.

Left moves to $(x-k+1, y-2)+(\overline{x+1}, y-2)$ and, by induction, Left wins.
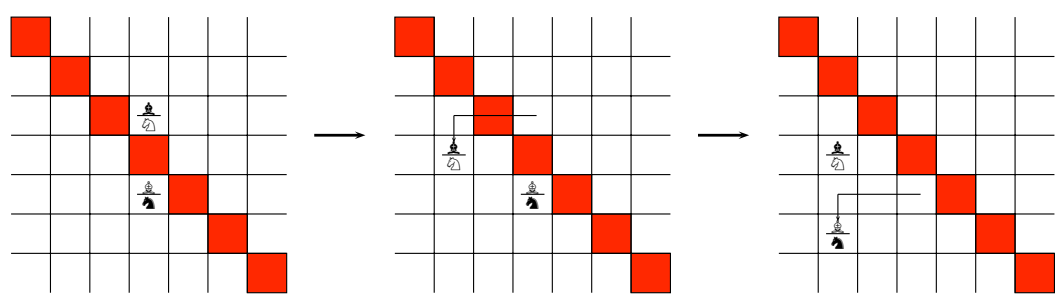

- Right plays to $(x-k-1, y-2)+\underline{(x, y)}$

Left moves to $(x-k-1, y-2)+(\overline{x-1}, y-2)$ and, by induction, Left wins.
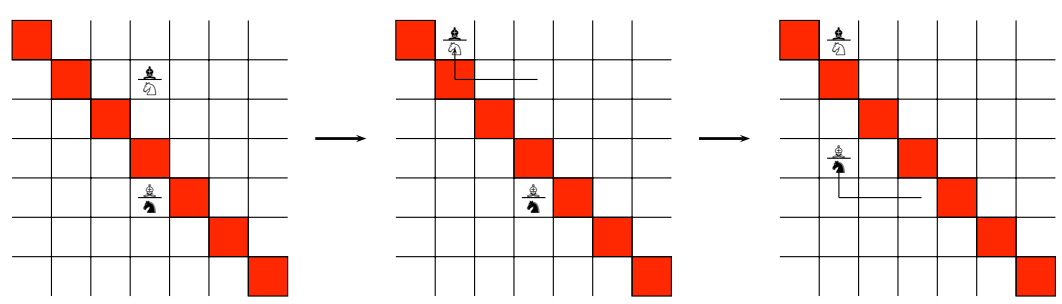

- Right plays to $(x-k-2, y+1)+(x, y)$

Left moves to $(x-k-1, y)+(x, y) \overline{\text { and }}$, by induction, Left wins. 

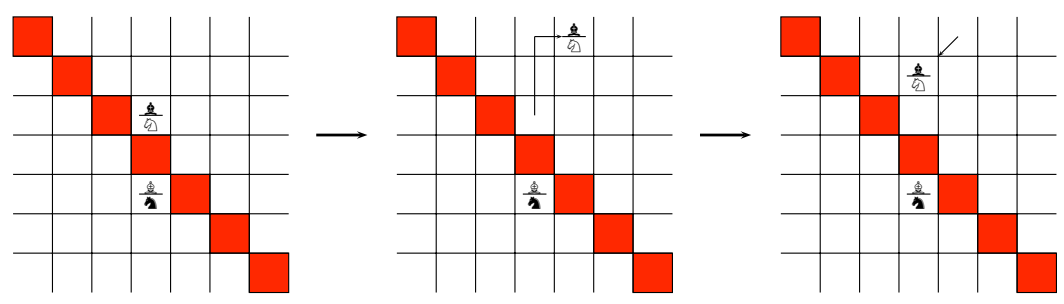

- Right plays to $(x-k-2, y-1)+(x, y)$ and $(x-1>y$ or $k=0)$. Left moves to $(x-k-2, y-1)+\underline{(\overline{x-2}, y-1)}$ and, by induction, Left wins.
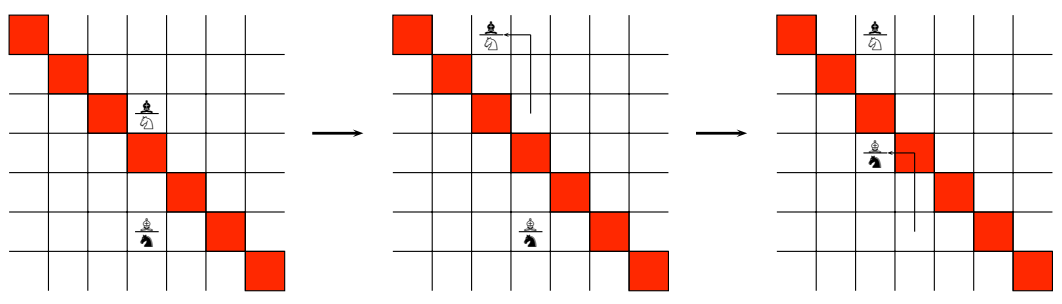

- Right plays to $(x-k-2, y-1)+(x, y)$ and, using the previous notation, $(x-k-2, y-1)$ is a red or a yellow cell.

Left moves to $(0, y-x+k+1)+(x, y)$ and, because $(0, y-x+k+1)=1$ or $(0, y-x+k+1)=\frac{1}{2}$ (Proposition 2 ), Left wins maintaining the second component below the principal diagonal.
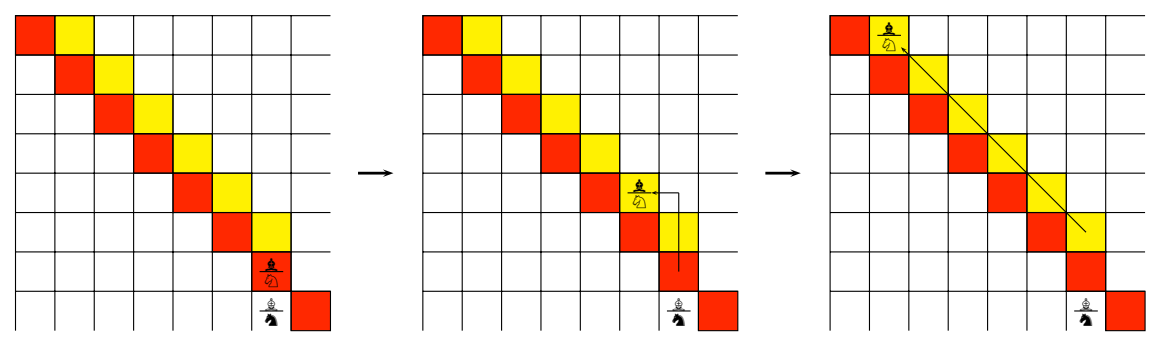

- Right plays to $(x-k-2, y-1)+(x, y)$ and $(x-k-2, y-1)$ is a green cell. Left moves to $(x-k-2, y-1)+\overline{(x-1}, y-2)$ and, if Right wants to avoid the induction, must move to $(x-k \overline{-4, y-2)+}(x-1, y-2)$. After this pair of moves, $(x-k-4, y-2)$ turns red or yellow and Left chooses the strategy of the previous item. 

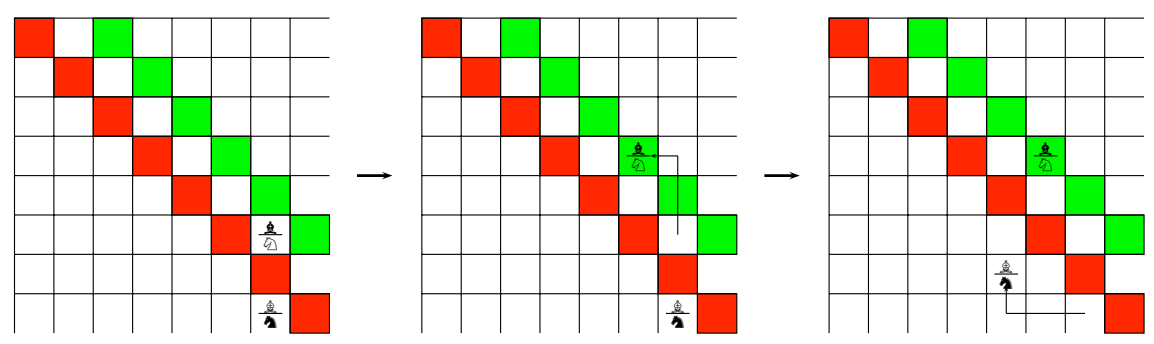

Proposition 4. If $x \geqslant 2$ then $(x, 1)=*$.

Proof. We can calculate by hand $(2,1)=*$. Now we prove the theorem by induction in $x$. The Left options of $(x, 1)$ are 0 (Proposition 1$)$. The Right options are $(x-2,0)=0$ and $(x-2,2)$. Against a Right's move to $(x-2,2)$, Left can immediately reply to $(x-1,1)$. By Proposition $3,(x-1,1) \geqslant(x, 1)$. So, by reversibility, the Right option $(x-2,2)$ can be replaced by Right options of $(x-1,1)$. But, by induction, $(x-1,1)=*$ and $(x-2,2)$ can be replaced by 0 .

Lemma 3. If $x>y$ then $1 \geqslant(x, y)$.

Proof. Let us analyze $1+(x, y)$ to see that Right, playing first, loses. Against a Right move (if he has one)

- To $1+\underline{\left(x^{\prime}, 0\right)}$. In that case, the game turned $1+0$.

- To $1+\underline{\left(x^{\prime}, 1\right)}$. In that case, the game turned $1 *$.

- To $1+\left(x^{\prime}, k\right)(k \geqslant 2)$. In that case, Left answers to $1+\left(x^{\prime}+1, k-2\right)$ reaching the same kind of position as before.

In all cases, Left wins.

Lemma 4. If $x>y$ then $(x-2, y+1) \geqslant(x+1, y-2)$.

Proof. Let us analyze $(x-2, y+1)+(x+1, y-2)$ to see that Right, playing first, loses. If Right plays in the component $(x-2, y+1)$, Left replies in the same component to the column $y-2$ and wins (Proposition 3 ).

If Right plays to $(x-2, y+1)+(x+1-i, y-2-i)$, Left replies to $(x-2-$ $i, y+1-i)+(x+1-i, y-2-i)$ maintaining the situation. If the Left answer was not available, that was because Right's move was to $(x-2, y+1)+(k, 1)(k \geqslant 2)$ or to $(x-2, y+1)+(k, 0)(k \geqslant 1)$. Against the first, Left moves the component $(x-2, y+1)$ to the column 1 and against the second, Left moves the component $(x-2, y+1)$ to the column 0 .

In both cases, Left wins. 
Theorem 1. The games $(x, y)$ for $x>y$ are all-small.

Proof. Let us consider $y \geqslant 2$ (the cases $y=0$ and $y=1$ are already known). By induction, Left options are all-small. Right has 4 options. By induction, $(x+1, y-2)$ and $(x-1, y-2)$ are all-small.

- Right option to $(x-2, y-1)$.

If $(x-2, y-1)$ is not in the principal diagonal, by induction, $(x-2, y-1)$ is all-small.

If $(x-2, y-1)$ is in the principal diagonal, Left can answer to $(1,1)=1$. By Lemma $3,1 \geqslant(x, y)$. So, the Right option is reversible to $\emptyset$.

- Right option to $(x-2, y+1)$.

By Lemma $4(x+1, y-2)$ dominates $(x-2, y+1)$. Because we are thinking for columns with index $y \geqslant 2,(x+1, y-2)$ is available.

\section{The General Recursive Process}

As we saw in the previous section, the Right option $(x-2, y+1)$ is dominated (see Theorem 1). For the sensible options, the column number is decreased by one or two. This strongly motivates the analysis of the recursion

$$
g(n)=\{g(0), \ldots g(n-1) \mid g(n-1), g(n-2)\} .
$$

This is a special case of a partizan subtraction game (see [4]). The first elements of the sequence are

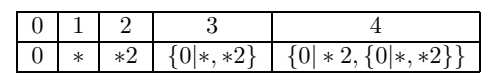

\begin{tabular}{|c|c|}
\hline 5 & 6 \\
\hline$\{0 \mid\{0 \mid *, * 2\},\{0 \mid * 2,\{0 \mid *, * 2\}\}\}$ & $\{0 \mid\{0 \mid * 2,\{0 \mid *, * 2\}\},\{0 \mid\{0 \mid *, * 2\},\{0 \mid * 2,\{0 \mid *, * 2\}\}\}\}$ \\
\hline
\end{tabular}

We can generalize the recursive law for similar chess knights (capable of making "larger" moves):

$$
g_{k}(n)=\left\{g_{k}(0), \ldots g_{k}(n-1) \mid g_{k}(n-k), g_{k}(n-2 k)\right\}(n \geqslant 0) .
$$

There is no problem with the $g_{k}(i)$ not previously defined. The empty set is available for the construction of the games.

For impartial subtraction games, it is well-known that $\operatorname{SUBTRACTION}\left(m s_{1}, \ldots, m s_{k}\right)$ is the $m$-plicate of $\operatorname{SUBTRACTION}\left(s_{1}, \ldots, s_{k}\right)$ ([2], page 98 and a proof in [5], page $36)$. We will prove that the general $g_{k}$ is also a kind of "dilation" of $g_{1}$. Just for intuition, we list the first elements of $g_{2}(n)$ and $g_{3}(n)$ : 


\begin{tabular}{|c|c|c|c|c|c|c|}
\hline 0 & 1 & 2 & 3 & 4 & 5 & 6 \\
\hline 0 & 1 & $\{1 \mid 0\}$ & $1 *$ & $\{1,1 * \mid 0,\{1 \mid 0\}\}$ & $1 * 2$ & $\{1 \mid\{1 \mid 0\},\{1,1 * \mid 0,\{1 \mid 0\}\}\}$ \\
\hline
\end{tabular}

\begin{tabular}{|c|c|}
\hline 7 & 8 \\
\hline$\{1 \mid 1 *, 1 * 2\}$ & $\{1 \mid\{1,1 * \mid 0,\{1 \mid 0\}\}\},\{1 \mid\{1 \mid 0\},\{1,1 * \mid 0,\{1 \mid 0\}\}\}\}$ \\
\hline
\end{tabular}

\begin{tabular}{|c|c|c|c|c|c|c|c|}
\hline 0 & 1 & 2 & 3 & 4 & 5 & 6 & 7 \\
\hline 0 & 1 & 2 & $\{2 \mid 0\}$ & $\{2 \mid 1\}$ & $2 *$ & $\{2,2 * \mid 0,\{2 \mid 0\}\}$ & $\{2,2 * \mid 1,\{2 \mid 1\}\}$ \\
\hline
\end{tabular}

\begin{tabular}{|c|c|c|}
\hline 8 & 9 & 10 \\
\hline $2 * 2$ & $\{2 \mid\{2 \mid 0\},\{2,2 * \mid 0,\{2 \mid 0\}\}\}$ & $\{2 \mid\{2 \mid 1\},\{2,2 * \mid 1,\{2 \mid 1\}\}\}$ \\
\hline
\end{tabular}

We start with a result about the left options of $g_{k}(n)$.

Lemma 5. For $k \geqslant 1$, we have

$$
\begin{gathered}
g_{k}(n)=\left\{g_{k}(0), \ldots, g_{k}(n-1) \mid g_{k}(n-k), g_{k}(n-2 k)\right\} \\
= \begin{cases}n & n \leqslant k-1 \\
\left\{k-1,(k-1) * \mid g_{k}(n-k), g_{k}(n-2 k)\right\} & 2 k \leqslant n \leqslant 3 k-1 \\
\left\{k-1 \mid g_{k}(n-k), g_{k}(n-2 k)\right\} & \text { other cases }\end{cases}
\end{gathered}
$$

Proof. Case (a) $n \leqslant k-1$. By definition,

$$
\begin{gathered}
g_{k}(0)=\{\mid\}=0 \\
g_{k}(1)=\left\{g_{k}(0) \mid\right\}=\{0 \mid\}=1 \\
(\ldots) \\
g_{k}(k-1)=\left\{g_{k}(k-2) \mid\right\}=\{k-2 \mid\}=k-1 .
\end{gathered}
$$

Case (b) $k \leqslant n \leqslant 2 k-1$. We already know that $g_{k}(0)=0, g_{k}(1)=1, \ldots$, $g_{k}(k-1)=k-1$. Therefore, by definition (and domination),

$$
\begin{gathered}
g_{k}(k)=\{k-1 \mid 0\} \\
g_{k}(k+1)=\{k-1,\{k-1 \mid 0\} \mid 1\} \\
g_{k}(k+2)=\{k-1,\{k-1 \mid 0\},\{k-1,\{k-1 \mid 0\} \mid 1\} \mid 2\}
\end{gathered}
$$

$(\vdots)$

We can use reversibility arguments: 


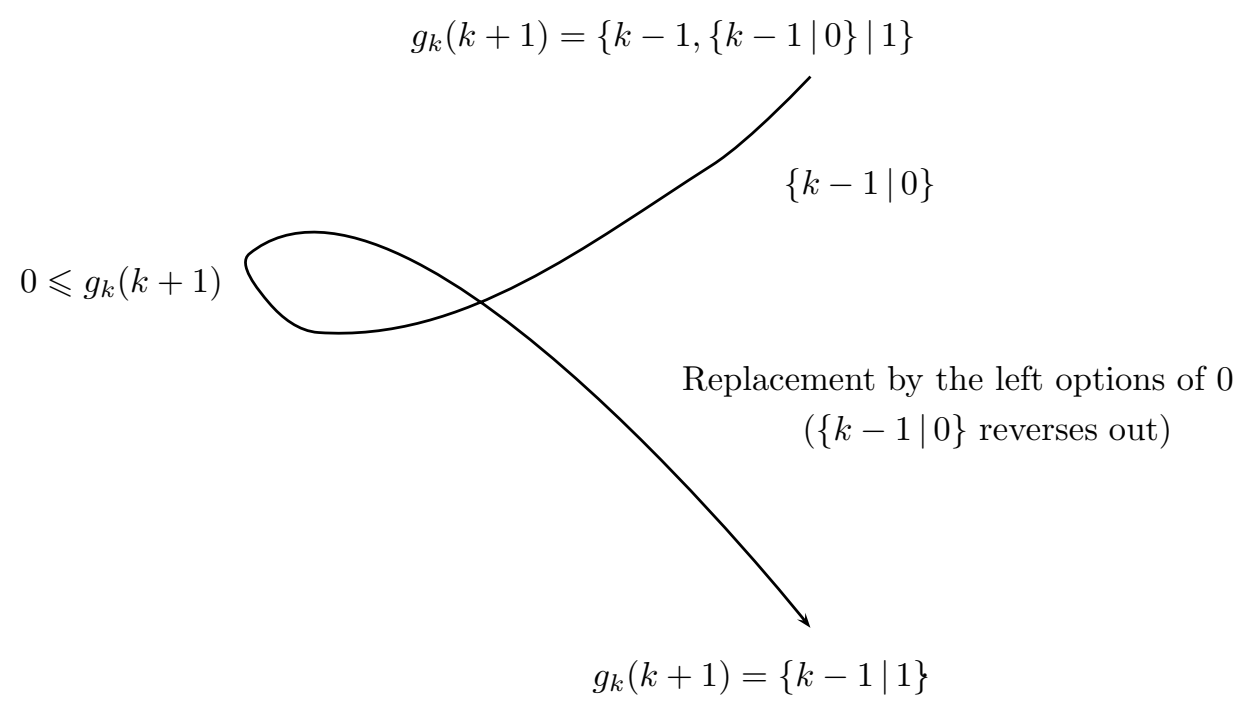

Similarly,

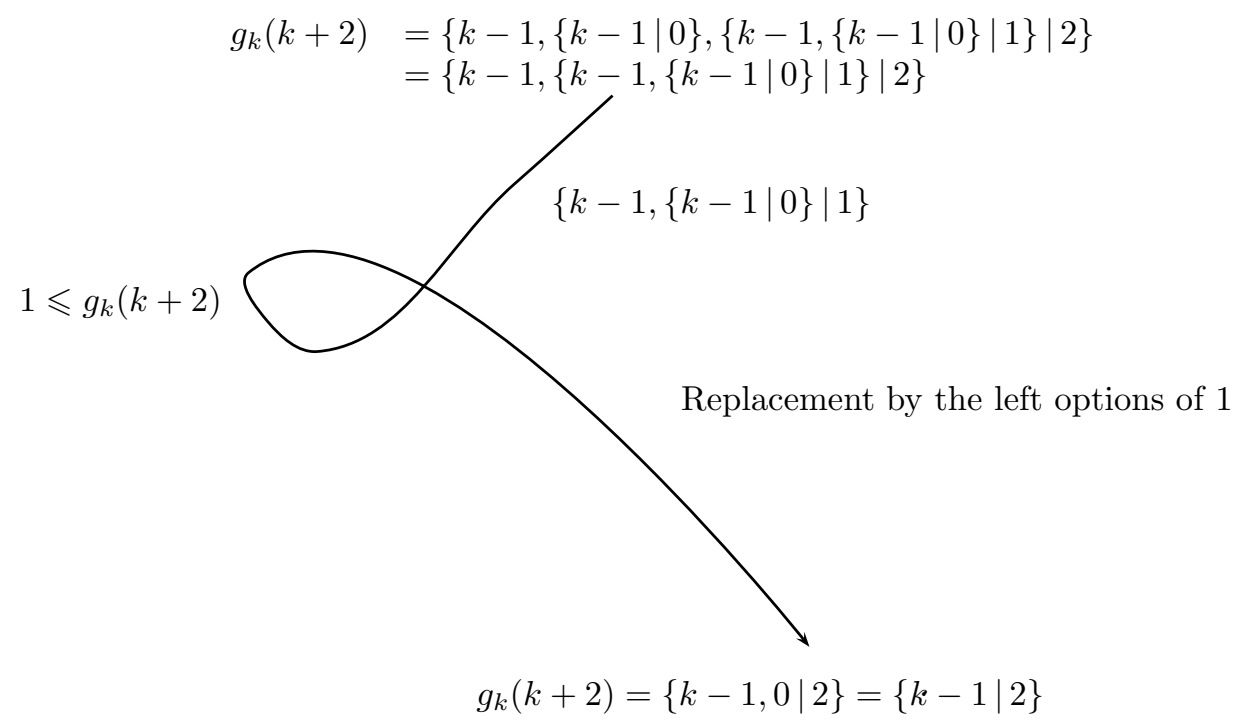

In general, for $0 \leqslant j \leqslant k-1$,

$$
g_{k}(k+j)=\left\{k-1, g_{k}(k), g_{k}(k+1), \ldots, g_{k}(k+j-1) \mid j\right\}
$$

and

$g_{k}(k)$ reverses out through 0 ;

$g_{k}(k+1)$ reverses through 1 to 0 which is dominated by $k-1$; 
$(\vdots)$ $g_{k}(k+j-1)$ reverses through $j-1$ to $j-2$ which is dominated by $k-1$.

The reversibility effects are justified by the inequality

$$
\left\{k-1, g_{k}(k), g_{k}(k+1), \ldots, g_{k}(k+j-1) \mid j\right\} \geqslant j-1
$$

We can conclude that the property is true for $k \leqslant n \leqslant 2 k-1$.

Case (c) $2 k \leqslant n \leqslant 3 k-1$. We have,

$$
\begin{gathered}
g_{k}(2 k)=\{k-1,(k-1) * \mid 0,\{k-1 \mid 0\}\} \\
\left.g_{k}(2 k+1)=\left\{k-1,(k-1) *, g_{k}(2 k) \mid 1,\{k-1 \mid 1\}\right\}\right\} \\
\left.g_{k}(2 k+2)=\left\{k-1,(k-1) *, g_{k}(2 k), g_{k}(2 k+1) \mid 2,\{k-1 \mid 2\}\right\}\right\}
\end{gathered}
$$

As the previous cases, it is easy to check that only the left options $k-1$ and $(k-1) *$ don't reverse. In fact, in general, for $0 \leqslant j \leqslant k-1$,

$g_{k}(2 k+j)=\left\{k-1,(k-1) *, g_{k}(2 k), g_{k}(2 k+1), \ldots, g_{k}(2 k+j-1) \mid j,\{k-1 \mid j\}\right\}$

and

$g_{k}(2 k)$ reverses out through 0 ;

$g_{k}(2 k+1)$ reverses through 1 to 0 which is dominated by $k-1$;

(:)

$g_{k}(2 k+j-1)$ reverses through $j-1$ to $j-2$ which is dominated by $k-1$.

The reversibility effects are justified by the inequality

$$
\left\{k-1,(k-1) *, g_{k}(2 k), g_{k}(2 k+1), \ldots, g_{k}(2 k+j-1) \mid j,\{k-1 \mid j\}\right\} \geqslant j-1 .
$$

We can conclude that the property is true for $2 k \leqslant n \leqslant 3 k-1$.

Case d) Other cases. In the other cases, also $(k-1) *$ reverses. This is true because, in these cases, we have

$$
\left\{k-1,(k-1) * \mid g_{k}(n-k), g_{k}(n-2 k)\right\} \geqslant k-1 .
$$

We can see that, in the game

$$
\left\{k-1,(k-1) * \mid g_{k}(n-k), g_{k}(n-2 k)\right\}+1-k,
$$

if Right begins, Right loses. This happens because the Left option $k-1$ is available in the games $g_{k}(n-k)$ and $g_{k}(n-2 k)$.

Now, we are able to prove a kind of "dilation" theorem. 
Theorem 2. Consider $n \geqslant 0$ and $k \geqslant 1$.

1. If $n \leqslant k-1, g_{k}(n)=n$.

2. If $n>k-1$, we obtain $g_{k}(n)$ from $g_{1}(n)$ as indicated: consider $i \in\{0, \ldots, k-$ $1\}$ such that $n \equiv i(\bmod k)$. Let $G$ be the game $g_{1}\left(\left\lfloor\frac{n}{k}\right\rfloor\right)$ (the form of the game according to its initial definition) and $J$ the game constructed from $G$ executing the following:

(a) Add $k-1$ to the games $G^{L}, G^{R L}, G^{R R L}, \ldots$

(b) Add $i$ to the games $G^{R}, G^{R R}, G^{R R R}, \ldots$ not affected by the first step.

We have $g_{k}(n)=J$.

Proof. The theorem is compatible with Lemma 5 because adding $k-1$ to the Left options of the game $g_{1}\left(\left\lfloor\frac{n}{k}\right\rfloor\right)$ generates exactly the same Left options for $g_{k}(n)$ indicated in the Lemma 5. So, we just have to analyze the Right options.

Just the induction step is non-trivial. Consider the game

$$
g_{k}(n+1)=\left\{g_{k}(0), \ldots, g_{k}(n) \mid g_{k}(n+1-k), g_{k}(n+1-2 k)\right\}
$$

By induction, we have to add $k-1$ and $i$ in the games $g_{1}\left(\left\lfloor\frac{n+1-k}{k}\right\rfloor\right)$ and $g_{1}\left(\left\lfloor\frac{n+1-2 k}{k}\right\rfloor\right)$ where $n+1-2 k \equiv n+1-k \equiv i(\bmod k)$.

But this is exactly the same as adding $k-1$ and $i$ in the Right options of $g_{1}\left(\left\lfloor\frac{n+1}{k}\right\rfloor\right)$. This is true because the Right options of $g_{1}\left(\left\lfloor\frac{n+1}{k}\right\rfloor\right)$ are $g_{1}\left(\left\lfloor\frac{n+1}{k}\right\rfloor-1\right)$ $=g_{1}\left(\left\lfloor\frac{n+1-k}{k}\right\rfloor\right)$ and $g_{1}\left(\left\lfloor\frac{n+1}{k}\right\rfloor-2\right)=g_{1}\left(\left\lfloor\frac{n+1-2 k}{k}\right\rfloor\right)$ and $n+1 \equiv i(\bmod k)$.

\section{References}

[1] M. H. Albert, R. J. Nowakowski, D. Wolfe, Lessons in Play: An Introduction to Combinatorial Game Theory, A. K. Peters, 2007.

[2] E. R. Berlekamp, J. Conway, R. Guy, Winning Ways. Academic Press, London, 1982.

[3] J. Conway, On Numbers and Games, Academic Press, 1976.

[4] A. S. Fraenkel, A. Kotzig. "Partizan Octal Games. Partizan Subtraction Games," International Journal of Game Theory, 1987.

[5] C. Santos, Some Notes on Impartial Games and Nim Dimension, PhD Thesis, University of Lisbon, 2010.

[6] W. A. Wythoff. A modification of the game of Nim, Niew Archief voor Wiskunde, 1907. 